\title{
AN ANALYTIC SOLUTION FOR THE FORCE BETWEEN TWO MAGNETIC DIPOLES
}

\author{
KAR W. YUNG, PETER B. LANDECKER* \\ and DANIEL D. VILLANI \\ Hughes Space and Communications Company, P.O. Box 92919, \\ Los Angeles, CA 90009, USA
}

(Received 7 February 1998; Accepted 30 April 1998)

\begin{abstract}
An analytic equation describing the force between two magnetic dipoles is derived in this paper. We assumed that the dipole sizes are small compared to their separation. A Taylor expansion for the first non-zero term was performed in order to derive the magnetic force analytic expression. Vector differential and path integral derivation approaches are used, and the same result is achieved with both methods. This force decreases with the fourth power of the distance between the dipoles. Accuracy estimates due to the Taylor approximation are given.
\end{abstract}

Keywords: Magnetic dipole, classical electrodynamic, magnetic force

\section{INTRODUCTION}

Since the forces between two magnetic dipoles are a function of spatial derivatives of their magnetic fields, it is extremely difficult to develop any physical intuition regarding the direction or magnitude of these forces, except in the cases where the dipole moments are either parallel or perpendicular to the separation vector between the dipoles. A closedform analytic expression would make it much easier to develop this intuition.

Forces on magnetic dipoles have been previously calculated by Greene and Karioris [1], Vaidman [2], Boyer [3], Brownstein [4],

* Corresponding author. 
Hrasko [5] and Hnizdo [6]. However, the force of one magnetic dipole on another has not yet been derived in electromagnetism textbooks or the periodical literature.

Such an analytic expression of this force is derived and presented in this paper for the case of dipole separation large compared with their size. Path integral and vector differentiation approaches are given here, with slightly different approximations used in each of these two cases. Both yielded the same closed-form result.

\section{FORCE BETWEEN MAGNETIC DIPOLES, PATH INTEGRAL APPROACH}

The magnetic force exerted by a circuit $a$ carrying a current $I_{a}$ on a circuit $b$ carrying a current $I_{b}$ (in MKS units) is given in Lorrain and Corson [7] as

$$
\vec{F}_{a b}=\frac{\mu_{0}}{4 \pi} I_{a} I_{b} \oint_{a} \oint_{b} \frac{\vec{d}_{b} \times\left(\vec{d}_{a} \times \vec{R}\right)}{R^{3}}
$$

where $\mu_{0}$ is the magnetic constant, equal to $4 \pi \times 10^{-7} \mathrm{NA}^{-2}, I_{a}$ the current of circuit $a, I_{b}$ the current of circuit $b, \vec{d}_{a}$ the path increment vector along circuit $a, \vec{d}_{b}$ the path increment vector along circuit $b$, and $\vec{R}$ the vector from circuit path element $\vec{d}_{a}$ to circuit path element $\vec{d}_{b}$. This geometry is illustrated in Fig. 1.

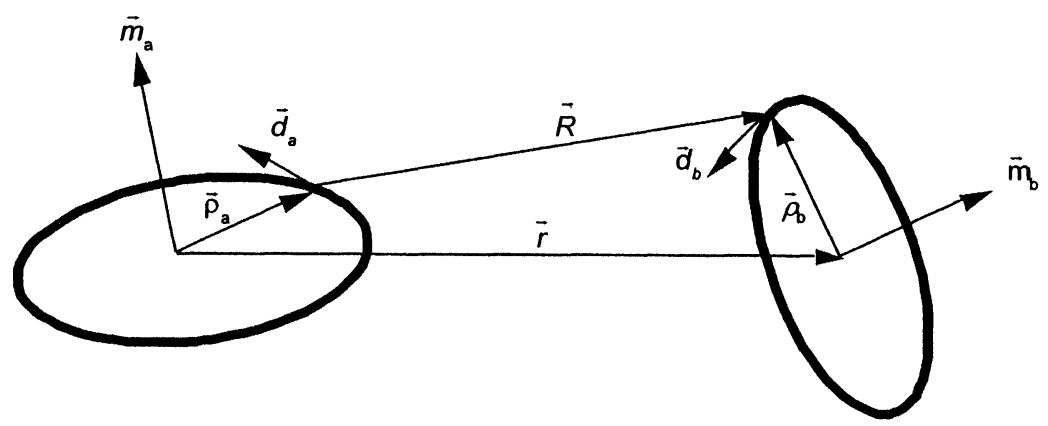

FIGURE 1 Geometry of the two magnetic dipoles. 
The magnetic dipole moments of these two circuits are defined as

$$
\vec{m}_{i}=\frac{I_{i}}{2} \oint \vec{\rho}_{i} \times \vec{d}_{i}, \quad i=a \text { or } b,
$$

where $\vec{\rho}_{a}$ is the vector from the center of the magnetic dipole $a$ to circuit element $\vec{d}_{a}$, and $\vec{\rho}_{b}$ the vector from the center of the magnetic dipole $b$ to circuit element $\vec{d}_{b}$.

We assume that the magnetic dipoles are planar circular circuits. The generic expression of the magnetic dipole moment then becomes

$$
\begin{aligned}
\vec{m} & =m \hat{m}=\frac{I}{2} \oint \vec{\rho} \times \vec{d}=\frac{I}{2} \oint \vec{\rho} \times(\rho \mathrm{d} \theta) \hat{d} \\
& =\left(\frac{I \rho^{2}}{2} \int_{0}^{2 \pi} \mathrm{d} \theta\right)(\hat{\rho} \times \hat{d})=\left(\pi \rho^{2} I\right)(\hat{\rho} \times \hat{d}),
\end{aligned}
$$

where $I$ is the current of the generic magnetic dipole circuit, $\hat{m}$ the unit dipole moment vector, $\hat{d}$ the unit path increment vector along the circuit, $\hat{\rho}$ the unit vector from the center of the magnetic dipole to the circuit path element $\hat{d}$ and $\theta$ the angle from an axis in the plane perpendicular to $\vec{m}$. This is illustrated in Fig. 2.

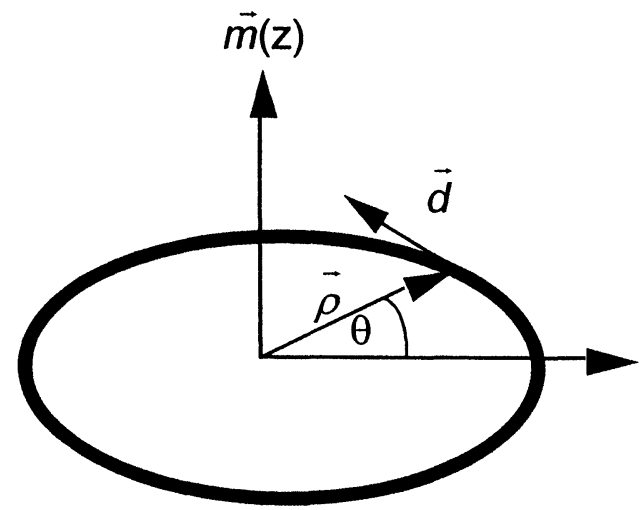

$(\mathrm{x})$

FIGURE 2 Geometry of the generic magnetic dipole moment circuit. 
We next express the three unit vectors $(\hat{m}, \hat{d}$, and $\hat{\rho})$ in terms of $\theta$ in the coordinate system shown in Fig. 2 as follows:

$$
\begin{gathered}
\hat{m}=\left(\begin{array}{l}
0 \\
0 \\
1
\end{array}\right)=\frac{\vec{m}}{m}, \\
\hat{\rho}=\left(\begin{array}{c}
\cos [\theta] \\
\sin [\theta] \\
0
\end{array}\right)=\frac{\vec{\rho}}{\rho},
\end{gathered}
$$

and

$$
\hat{d}=\left(\begin{array}{c}
-\sin [\theta] \\
\cos [\theta] \\
0
\end{array}\right)=\frac{\vec{d}}{\rho \mathrm{d} \theta} .
$$

Note that:

$$
\hat{\rho} \cdot \hat{d}=\hat{\rho} \cdot \hat{m}=\hat{d} \cdot \hat{m}=0,
$$

and

$$
\int \hat{\rho} \mathrm{d} \theta=\int \hat{d} \mathrm{~d} \theta=\left(\begin{array}{l}
0 \\
0 \\
0
\end{array}\right)=\overrightarrow{0} .
$$

We next consider a general vector $\vec{v}$ in the coordinate system of a circular magnetic dipole. We derive the following relations using Eqs. (4)-(6) as follows:

$$
\begin{gathered}
\vec{v}=\left(\begin{array}{l}
x \\
y \\
z
\end{array}\right), \\
\int \vec{v} \cdot \hat{m} \mathrm{~d} \theta=2 \pi \vec{v} \cdot \hat{m}, \\
\int \vec{v} \cdot \hat{\rho} \mathrm{d} \theta=\int(x \cos [\theta]+y \sin [\theta]) \mathrm{d} \theta=0,
\end{gathered}
$$




$$
\begin{gathered}
\int \vec{v} \cdot \hat{d} \mathrm{~d} \theta=\int(-x \sin [\theta]+y \cos [\theta]) \mathrm{d} \theta=0 \\
\left.\int \hat{d}(\vec{v} \cdot \hat{\rho}) \mathrm{d} \theta=\int\left(\begin{array}{c}
-y \sin ^{2}[\theta] \\
x \cos ^{2}[\theta] \\
0
\end{array}\right)+\sin [\theta] \cos [\theta]\left(\begin{array}{c}
-x \\
y \\
0
\end{array}\right)\right) \mathrm{d} \theta \\
=\pi\left(\begin{array}{c}
-y \\
x \\
0
\end{array}\right)=\pi \hat{m} \times \vec{v}, \\
\int \hat{d}(\vec{v} \cdot \hat{d}) \mathrm{d} \theta=\pi\left(\begin{array}{c}
x \\
y \\
0
\end{array}\right)=\pi(\vec{v}-\hat{m}(\hat{m} \cdot \vec{v})), \\
\int \hat{\rho}(\vec{v} \cdot \hat{\rho}) \mathrm{d} \theta=\pi(\vec{v}-\hat{m}(\hat{m} \cdot \vec{v}))
\end{gathered}
$$

and

$$
\int \hat{\rho}(\vec{v} \cdot \hat{d}) \mathrm{d} \theta=-\pi \hat{m} \times \vec{v}
$$

In the expansion below, we will be evaluating integrals of certain vector products. Before we get to those specific integrals, it is useful to examine some shortcuts that allow us to express these integrals of products in terms of products of vectors and the magnetic moment. Consider now two general vectors $\vec{g}$ and $\vec{h}$ in the coordinate system of one of the circular magnetic dipoles. We derive the following relations using Eqs. (4)-(6):

$$
\begin{aligned}
& \vec{g}=\left(\begin{array}{l}
x_{g} \\
y_{g} \\
z_{g}
\end{array}\right), \\
& \vec{h}=\left(\begin{array}{l}
x_{h} \\
y_{h} \\
z_{h}
\end{array}\right),
\end{aligned}
$$




$$
\begin{aligned}
& \int(\vec{g} \cdot \hat{\rho})(\vec{h} \cdot \hat{d}) \mathrm{d} \theta=\int\left(x_{g} \cos [\theta]+y_{g} \sin [\theta]\right)\left(-x_{h} \sin [\theta]+y_{h} \cos [\theta]\right) \mathrm{d} \theta \\
& =\pi\left(x_{g} y_{h}-y_{g} x_{h}\right) \\
& =\pi(\vec{g} \times \vec{h}) \hat{m}, \\
& \int(\vec{g} \cdot \hat{\rho})(\vec{h} \cdot \hat{\rho}) \mathrm{d} \theta=\pi(\vec{g} \cdot \vec{h}-(\vec{g} \cdot \hat{m})(\vec{h} \cdot \hat{m}))=\pi(\vec{g} \times \hat{m}) \cdot(\vec{h} \times \hat{m}), \\
& \int(\vec{g} \cdot \hat{d})(\vec{h} \cdot \hat{d}) \mathrm{d} \theta=\pi(\vec{g} \cdot \vec{h}-(\vec{g} \cdot \hat{m})(\vec{h} \cdot \hat{m}))=\pi(\vec{g} \times \hat{m}) \cdot(\vec{h} \times \hat{m}), \\
& \int(\vec{g} \cdot \hat{\rho})(\vec{h} \times \hat{d}) \mathrm{d} \theta=\int(\vec{g} \cdot \hat{\rho})(\vec{h} \times(\hat{m} \times \hat{\rho})) \mathrm{d} \theta \\
& =\int(\vec{g} \cdot \hat{\rho})(\hat{m}(\vec{h} \cdot \hat{\rho})-\hat{\rho}(\vec{h} \cdot \hat{m})) \mathrm{d} \theta \\
& =\pi(\hat{m} \vec{g} \cdot \vec{h}-\vec{g}(\vec{h} \cdot \hat{m}))=\pi(\vec{g} \times \hat{m}) \times \vec{h}, \\
& \int(\vec{g} \cdot \hat{d})(\vec{h} \times \hat{\rho}) \mathrm{d} \theta=-\pi(\hat{m} \vec{g} \cdot \vec{h}-\vec{g}(\vec{h} \cdot \hat{m}))=-\pi(\vec{g} \times \hat{m}) \times \vec{h} .
\end{aligned}
$$

For the far field approximation, we assume that the separation vector $\vec{R}$ between the dipoles is much larger than the dimensions of the dipoles. In effect, we are using a Taylor expansion and ignoring the higher order terms of $r^{-1}$. That is,

$$
\begin{gathered}
\vec{R}=\vec{r}+\Delta \vec{r}=\vec{r}+\left(\vec{\rho}_{b}-\vec{\rho}_{a}\right), \\
R^{2}=r^{2}+2 \vec{r} \cdot \Delta \vec{r}+\Delta \vec{r} \cdot \Delta \vec{r}=r^{2}\left(1+2 \frac{\hat{r} \cdot \Delta \vec{r}}{r}+\frac{\Delta \vec{r} \cdot \Delta \vec{r}}{r^{2}}\right),
\end{gathered}
$$

and

$$
\frac{1}{R^{3}} \approx \frac{1}{r^{3}}\left(1-3 \frac{\hat{r} \cdot \Delta \vec{r}}{r}-\frac{3}{2} \frac{\Delta \vec{r} \cdot \Delta \vec{r}}{r^{2}}+\frac{15}{2} \frac{(\hat{r} \cdot \Delta \vec{r})^{2}}{r^{2}}\right),
$$

where $\vec{r}$ is the vector from the center of magnetic dipole $a$ to the center of dipole $b, \hat{r}$ the unit vector along $\vec{r}$, and $\Delta \vec{r}$ is the difference of center to circuit vectors and equals $\vec{\rho}_{b}-\vec{\rho}_{a}$. 
We substitute the above expansion (26) into the integrand of the force equation (1) as follows:

$$
\begin{aligned}
& \frac{\vec{d}_{b} \times\left(\vec{d}_{a} \times \vec{R}\right)}{R^{3}} \\
& \quad \frac{\vec{d}_{b} \times\left(\vec{d}_{a} \times \vec{R}\right)}{r^{3}}\left(1-3 \frac{\hat{r} \cdot \Delta \vec{r}}{r}-\frac{3}{2} \frac{\Delta \vec{r} \cdot \Delta \vec{r}}{r^{2}}+\frac{15}{2} \frac{(\hat{r} \cdot \Delta \vec{r})^{2}}{r^{2}}\right) .
\end{aligned}
$$

We ignore the first term whose integral is zero. We then substitute expressions (7) and (24) into Eq. (27). The integrand of the force equation in the far field therefore becomes:

$$
\begin{aligned}
\frac{\vec{d}_{b} \times\left(\vec{d}_{a} \times \vec{R}\right)}{r^{3}}\left(-3 \frac{\hat{r} \cdot \Delta \vec{r}}{r}-\frac{3}{2} \frac{\Delta \vec{r} \cdot \Delta \vec{r}}{r^{2}}+\frac{15}{2} \frac{(\hat{r} \cdot \Delta \vec{r})^{2}}{r^{2}}\right) \\
=\frac{\vec{d}_{b} \times\left(\vec{d}_{a} \times\left(\vec{r}+\vec{\rho}_{b}-\vec{\rho}_{a}\right)\right)}{r^{4}}\left(-3 \hat{r} \cdot \Delta \vec{r}-\frac{3}{2} \frac{\Delta \vec{r} \cdot \Delta \vec{r}}{r}+\frac{15}{2} \frac{(\hat{r} \cdot \Delta \vec{r})^{2}}{r}\right) \\
=\frac{1}{r^{4}}\left(\vec{d}_{a}\left(\vec{d}_{b} \cdot \vec{r}\right)-\vec{r}\left(\vec{d}_{a} \cdot \vec{d}_{b}\right)-\vec{\rho}_{b}\left(\vec{d}_{a} \cdot \vec{d}_{b}\right)-\vec{d}_{a}\left(\vec{d}_{b} \cdot \vec{\rho}_{a}\right)+\vec{\rho}_{a}\left(\vec{d}_{b} \cdot \vec{d}_{a}\right)\right) \\
\quad \times\left(-3 \hat{r} \cdot\left(\vec{\rho}_{b}-\vec{\rho}_{a}\right)-\frac{3}{2} \frac{\left(\vec{\rho}_{b}-\vec{\rho}_{a}\right)^{2}}{r}+\frac{15}{2} \frac{\left(\hat{r} \cdot \vec{\rho}_{b}-\hat{r} \cdot \vec{\rho}_{a}\right)^{2}}{r}\right) .
\end{aligned}
$$

Some of the terms in the above expression have zero integrals as indicated by Eq. (8), (11), and (12). We therefore ignore these terms and now have:

$$
\begin{aligned}
\frac{\vec{d}_{b} \times\left(\vec{d}_{a} \times \vec{R}\right)}{r^{3}}\left(-3 \frac{\hat{r} \cdot \Delta \vec{r}}{r}-\frac{3}{2} \frac{\Delta \vec{r} \cdot \Delta \vec{r}}{r^{2}}+\frac{15}{2} \frac{(\hat{r} \cdot \Delta \vec{r})^{2}}{r^{2}}\right) \\
=\frac{1}{r^{4}}\left(\left(\vec{d}_{a}\left(\vec{d}_{b} \cdot \hat{r}\right)-\hat{r}\left(\vec{d}_{a} \cdot \vec{d}_{b}\right)\right)\left(3\left(\vec{\rho}_{a} \cdot \vec{\rho}_{b}\right)-15\left(\hat{r} \cdot \vec{\rho}_{a}\right)\left(\hat{r} \cdot \vec{\rho}_{b}\right)\right)\right) \\
\quad+3 \vec{\rho}_{b}\left(\vec{d}_{a} \cdot \vec{d}_{b}\right)\left(\hat{r} \cdot \vec{\rho}_{b}\right)+3\left(-\vec{d}_{a}\left(\vec{d}_{b} \cdot \vec{\rho}_{a}\right)+\vec{\rho}_{a}\left(\vec{d}_{b} \cdot \vec{d}_{a}\right)\right)\left(\hat{r} \cdot \vec{\rho}_{a}\right) .
\end{aligned}
$$

We then evaluate integrals of these terms with Eqs. (10)-(16) and (18)-(23) as follows:

$$
\begin{aligned}
\oint_{b} \oint_{a} \vec{d}_{a}\left(\vec{d}_{b} \cdot \hat{r}\right)\left(\vec{\rho}_{b} \cdot \vec{\rho}_{a}\right) & =\rho_{a}^{2} \rho_{b}^{2} \int\left(\hat{d}_{b} \cdot \hat{r}\right) \int \hat{d}_{a}\left(\hat{\rho}_{a} \cdot \hat{\rho}_{b}\right) \mathrm{d} \theta_{a} \mathrm{~d} \theta_{b} \\
& =\frac{m_{a} m_{b}}{I_{a} I_{b}}\left(-\left(\hat{r} \cdot \hat{m}_{a}\right) \hat{m}_{b}+\left(\hat{m}_{a} \cdot \hat{m}_{b}\right) \hat{r}\right)
\end{aligned}
$$




$$
\begin{aligned}
& -\oint_{b} \oint_{a} \hat{r}\left(\vec{d}_{a} \cdot \vec{d}_{b}\right)\left(\vec{\rho}_{b} \cdot \vec{\rho}_{a}\right) \\
& =-\hat{r} \rho_{a}^{2} \rho_{b}^{2} \iint\left(\hat{d}_{a} \cdot \hat{d}_{b}\right)\left(\hat{\rho}_{a} \cdot \hat{\rho}_{b}\right) \mathrm{d} \theta_{a} \mathrm{~d} \theta_{b}=-\frac{2 m_{a} m_{b}}{I_{a} I_{b}}\left(\hat{m}_{a} \cdot \hat{m}_{b}\right) \hat{r} \\
& -5 \oint_{b} \oint_{a} \vec{d}_{a}\left(\vec{d}_{b} \cdot \hat{r}\right)\left(\hat{r} \cdot \vec{\rho}_{a}\right)\left(\hat{r} \cdot \vec{\rho}_{b}\right) \\
& =-5 \rho_{a}^{2} \rho_{b}^{2} \int\left(\hat{d}_{b} \cdot \hat{r}\right)\left(\hat{r} \cdot \hat{\rho}_{b}\right) \int \hat{d}_{a}\left(\vec{r} \cdot \hat{\rho}_{a}\right) \mathrm{d} \theta_{a} \mathrm{~d} \theta_{b}=0 \\
& 5 \oint_{b} \oint_{a} \hat{r}\left(\vec{d}_{a} \cdot \vec{d}_{b}\right)\left(\hat{r} \cdot \vec{\rho}_{a}\right)\left(\hat{r} \cdot \vec{\rho}_{b}\right)=5 \rho_{a}^{2} \rho_{b}^{2} \int\left(\hat{r} \cdot \hat{\rho}_{b}\right) \\
& \times \int\left(\hat{d}_{a} \cdot \hat{d}_{b}\right)\left(\hat{r} \cdot \hat{\rho}_{a}\right) \mathrm{d} \theta_{a} \mathrm{~d} \theta_{b} \\
& =5 \frac{m_{a} m_{b}}{I_{a} I_{b}} \hat{r}\left(\hat{m}_{a} \cdot \hat{m}_{b}-\left(\vec{r} \cdot \hat{m}_{a}\right)\left(\vec{r} \cdot \hat{m}_{b}\right)\right), \\
& -\oint_{b} \oint_{a} \vec{\rho}_{b}\left(\vec{d}_{b} \cdot \vec{d}_{a}\right)\left(\hat{r} \cdot \vec{\rho}_{a}\right)=-\rho_{a}^{2} \rho_{b}^{2} \int \hat{\rho}_{b} \int\left(\hat{d}_{b} \cdot \hat{d}_{a}\right)\left(\hat{r} \cdot \hat{\rho}_{a}\right) \mathrm{d} \theta_{a} \mathrm{~d} \theta_{b} \\
& =\frac{m_{a} m_{b}}{I_{a} I_{b}}\left(\left(\hat{m}_{b} \cdot \hat{r}\right) \hat{m}_{a}-\left(\hat{m}_{b} \cdot \hat{m}_{a}\right) \hat{r}\right), \\
& \oint_{b} \oint_{a} \vec{d}_{a}\left(\vec{d}_{b} \cdot \vec{\rho}_{a}\right)\left(\hat{r} \cdot \vec{\rho}_{b}\right)=\rho_{a}^{2} \rho_{b}^{2} \int\left(\hat{r} \cdot \hat{\rho}_{b}\right) \int \hat{d}_{a}\left(\hat{d}_{b} \cdot \hat{\rho}_{a}\right) \mathrm{d} \theta_{a} \mathrm{~d} \theta_{b} \\
& =\frac{m_{a} m_{b}}{I_{a} I_{b}}\left(\left(\hat{r} \cdot \hat{m}_{a}\right) \hat{m}_{b}-\left(\hat{m}_{a} \cdot \hat{m}_{b}\right) \hat{r}\right), \\
& -\oint_{b} \oint_{a} \vec{\rho}_{a}\left(\vec{d}_{b} \cdot \vec{d}_{a}\right)\left(\hat{r} \cdot \vec{\rho}_{b}\right)=-\rho_{a}^{2} \rho_{b}^{2} \int\left(\hat{r} \cdot \hat{\rho}_{b}\right) \int \hat{\rho}_{a}\left(\hat{d}_{b} \cdot \hat{d}_{a}\right) \mathrm{d} \theta_{a} \mathrm{~d} \theta_{b} \\
& =\frac{m_{a} m_{b}}{I_{a} I_{b}}\left(\left(\hat{r} \cdot \hat{m}_{a}\right) \hat{m}_{b}-\left(\hat{m}_{a} \cdot \hat{m}_{b}\right) \hat{r}\right) .
\end{aligned}
$$

Using results in Eqs. (28)-(36), the final force equation of dipole $a$ on dipole $b$ after path integration becomes:

$$
\begin{aligned}
\vec{F}_{a b}= & \frac{3 \mu_{0} m_{a} m_{b}}{4 \pi r^{4}}\left(\hat{r}\left(\hat{m}_{a} \cdot \hat{m}_{b}\right)+\hat{m}_{a}\left(\hat{r} \cdot \hat{m}_{b}\right)+\hat{m}_{b}\left(\hat{r} \cdot \hat{m}_{a}\right)\right. \\
& \left.-5 \hat{r}\left(\hat{r} \cdot \hat{m}_{a}\right)\left(\hat{r} \cdot \hat{m}_{b}\right)\right) \\
= & \frac{3 \mu_{0}}{4 \pi r^{4}}\left(\left(\hat{r} \times \hat{m}_{a}\right) \times \hat{m}_{b}+\left(\hat{r} \times \hat{m}_{b}\right) \times \hat{m}_{a}-2 \hat{r}\left(\vec{m}_{a} \cdot \vec{m}_{b}\right)\right. \\
& \left.+5 \hat{r}\left(\left(\hat{r} \times \vec{m}_{a}\right) \cdot\left(\hat{r} \times \vec{m}_{b}\right)\right)\right) .
\end{aligned}
$$




\section{FORCE BETWEEN DIPOLES, VECTOR}

\section{DIFFERENTIATION APPROACH}

We can also derive the force expression with the vector differentiation approach rather than the path integral approach shown above. The magnetic field generated by dipole $a$ at the location of dipole $b$ is given by:

$$
\vec{B}_{a b}=-\frac{\mu_{0}}{4 \pi} \vec{\nabla} \frac{\vec{m}_{a} \cdot \vec{r}}{r^{3}}
$$

The force exerted by dipole $a$ on dipole $b$ can be derived from potential energy considerations as follows:

$$
\vec{F}_{a b}=-\vec{\nabla}\left(-\vec{B}_{a b} \cdot \vec{m}_{b}\right)=\vec{\nabla}\left(\vec{B}_{a b} \cdot \vec{m}_{b}\right)=-\frac{\mu_{0}}{4 \pi} \vec{\nabla}\left(\left(\vec{\nabla} \frac{\vec{m}_{a} \cdot \vec{r}}{r^{3}}\right) \cdot \vec{m}_{b}\right) .
$$

Forces shown above can be simplified. First, let us look at gradients of the following functions:

$$
\vec{\nabla} \frac{1}{r^{n}}=\left(\begin{array}{c}
\partial / \partial x \\
\partial / \partial y \\
\partial / \partial z
\end{array}\right) \frac{1}{r^{n}}=-\frac{n}{r^{n+1}}\left(\begin{array}{c}
x / r \\
y / r \\
z / r
\end{array}\right)=-\frac{n \vec{r}}{r^{n+2}}
$$

and

$$
\begin{aligned}
\vec{\nabla}\left(\vec{v}_{1} \cdot \vec{r}\right)=\left(\begin{array}{c}
\partial / \partial x \\
\partial / \partial y \\
\partial / \partial z
\end{array}\right)\left(\vec{v}_{1} \cdot \vec{r}\right) & =\left(\begin{array}{l}
\partial / \partial x\left(x_{1} x+y_{1} y+z_{1} z\right) \\
\partial / \partial y\left(x_{1} x+y_{1} y+z_{1} z\right) \\
\partial / \partial z\left(x_{1} x+y_{1} y+z_{1} z\right)
\end{array}\right) \\
& =\left(\begin{array}{l}
x_{1} \\
y_{1} \\
z_{1}
\end{array}\right)=\vec{v}_{1} .
\end{aligned}
$$

These relations can be used to simplify Eq. (39) as follows:

$$
\begin{aligned}
\vec{F}_{a b} & =-\frac{\mu_{0}}{4 \pi} \vec{\nabla}\left(\left(\vec{\nabla} \frac{\vec{m}_{a} \cdot \vec{r}}{r^{3}}\right) \cdot \vec{m}_{b}\right) \\
& =-\frac{\mu_{0}}{4 \pi} \vec{\nabla}\left(\left(\frac{1}{r^{3}} \vec{\nabla}\left(\vec{m}_{a} \cdot \vec{r}\right)+\left(\vec{m}_{a} \cdot \vec{r}\right) \vec{\nabla} \frac{1}{r^{3}}\right) \cdot \vec{m}_{b}\right) \\
& =-\frac{\mu_{0}}{4 \pi} \vec{\nabla}\left(\left(\frac{\vec{m}_{a}}{r^{3}}-\left(\vec{m}_{a} \cdot \vec{r}\right) \frac{3 \vec{r}}{r^{5}}\right) \cdot \vec{m}_{b}\right)
\end{aligned}
$$




$$
\begin{aligned}
= & -\frac{\mu_{0}}{4 \pi} \vec{\nabla}\left(\frac{\vec{m}_{a} \cdot \vec{m}_{b}}{r^{3}}-3 \frac{\left(\vec{m}_{a} \cdot \vec{r}\right)\left(\vec{m}_{b} \cdot \vec{r}\right)}{r^{5}}\right) \\
= & -\frac{\mu_{0}}{4 \pi}\left(\left(\vec{m}_{a} \cdot \vec{m}_{b}\right) \vec{\nabla} \frac{1}{r^{3}}-3\left(\vec{m}_{a} \cdot \vec{r}\right)\left(\vec{m}_{b} \cdot \vec{r}\right) \vec{\nabla} \frac{1}{r^{5}}\right. \\
& \left.-3 \frac{\left(\vec{m}_{b} \cdot \vec{r}\right)}{r^{5}} \vec{\nabla}\left(\vec{m}_{a} \cdot \vec{r}\right)-3 \frac{\left(\vec{m}_{a} \cdot \vec{r}\right)}{r^{5}} \vec{\nabla}\left(\vec{m}_{b} \cdot \vec{r}\right)\right) \\
= & \frac{3 \mu_{0} m_{a} m_{b}}{4 \pi r^{4}}\left(\hat{r}\left(\hat{m}_{a} \cdot \hat{m}_{b}\right)+\hat{m}_{a}\left(\hat{r} \cdot \hat{m}_{b}\right)+\hat{m}_{b}\left(\hat{r} \cdot \hat{m}_{a}\right)\right. \\
& \left.-5 \hat{r}\left(\hat{r} \cdot \hat{m}_{a}\right)\left(\hat{r} \cdot \hat{m}_{b}\right)\right) \\
= & \frac{3 \mu_{0}}{4 \pi r^{4}}\left(\left(\hat{r} \times \vec{m}_{a}\right) \times \vec{m}_{b}+\left(\hat{r} \times \vec{m}_{b}\right) \times \vec{m}_{a}-2 \hat{r}\left(\vec{m}_{a} \cdot \vec{m}_{b}\right)\right. \\
& \left.+5 \hat{r}\left(\left(\hat{r} \times \vec{m}_{a}\right) \cdot\left(\hat{r} \times \vec{m}_{b}\right)\right)\right) .
\end{aligned}
$$

Note that Eq. (37) and (42) are identical. In addition, for magnetic dipoles aligned in the same direction, perpendicular to their separation direction, a pure repulsive force results. For the case of two magnetic dipoles oriented in opposite directions, again both perpendicular to their separation direction, a pure attraction force results. For the case of the magnetic dipoles and their separation orientation direction all coaligned, a pure attractive force results. For other combinations of orientations, both forces and torques can be produced. In a companion paper, torques between two magnetic dipoles are discussed [8]. Note also that these equations allow us to understand the forces in fairly simple and intuitive terms. Here, the total force is proportional to the product of the two magnetic moments, and inversely proportional to the fourth power of the distance between them. There is a component parallel to the separation vector, and a component parallel to each dipole moment. The relative magnitudes of these components is a function of the geometrical relationships between the two dipole moments and between each dipole and the separation vector between them.

In addition, we computed magnetic forces for several different cases with our derived formula (42). We then compare these results with those obtained with numerical integration of the original exact Eq. (1). We refer to the results derived with our formula as the "Formula" results 
and those with numerical integration of the exact equation as the "Exact" results. We have varied the numerical integration step size to ensure contribution of the numerical step error is insignificant in our analysis.

We assume magnetic moments for $a$ and $b$ both equal to $100 \mathrm{~A} \mathrm{~m}^{2}$, the radius of the magnetic dipole loop is $10 \mathrm{~cm}$, and a spacing of $1 \mathrm{~m}$ between the two dipoles. We considered four different dipole orientations in a coordinate system with the $x$-axis pointing along $\hat{r}$, the separation vector from $a$ to $b$. The directions of the unit magnetic moment vectors and force vectors computed with "Formula" and "Exact" approaches are presented as shown in Table I.

These results demonstrate that our formula is very good when the separation distance $r$ is large compared to the magnetic moment radius $\rho$. We analyzed the sensitivity of this Taylor expansion error contribution by computing the ratio of force magnitudes derived with the "Exact" and "Formula" approaches. We plot this ratio versus $r / \rho$ for the four different cases discussed above. Results are presented in Figs. 3-6.

The results show when $r / \rho$ is about 7, our formula is well within $10 \%$ of the exact formula.

TABLE I

\begin{tabular}{lcccc}
\hline & \multicolumn{4}{c}{ CASE } \\
\cline { 2 - 4 }$\hat{m}_{a}$ & I & II & III & IV \\
\hline & $\left(\begin{array}{l}1 \\
0 \\
0\end{array}\right)$ & $\left(\begin{array}{c}0 \\
-1 \\
0\end{array}\right)$ & $\left(\begin{array}{c}1 / \sqrt{2} \\
0 \\
1 / \sqrt{2}\end{array}\right)$ & $\left(\begin{array}{l}0 \\
0 \\
1\end{array}\right)$ \\
$\hat{m}_{b}$ & $\left(\begin{array}{l}1 \\
0 \\
0\end{array}\right)$ & $\left(\begin{array}{l}1 \\
0 \\
0\end{array}\right)$ & $\left(\begin{array}{l}0 \\
0 \\
1\end{array}\right)$ & $\left(\begin{array}{l}0 \\
0 \\
1\end{array}\right)$ \\
Formula $\vec{F}_{a b}(\mathrm{~N})$ & $\left(\begin{array}{c}-0.006 \\
0 \\
0\end{array}\right)$ & $\left(\begin{array}{c}0 \\
-0.003 \\
0\end{array}\right)$ & $\left(\begin{array}{c}0.003 / \sqrt{2} \\
0 \\
0.003 / \sqrt{2}\end{array}\right)$ & $\left(\begin{array}{c}0.003 \\
0 \\
0\end{array}\right)$ \\
Exact $\vec{F}_{a b}(\mathrm{~N})$ & $\left(\begin{array}{c}-0.0057126 \\
0 \\
0\end{array}\right)$ & $\left(\begin{array}{c}0.002099 \\
0 \\
-0.00298 \\
0\end{array}\right)$ & $\left(\begin{array}{c}0.003117 \\
0 \\
0.002196\end{array}\right)$ & \\
\hline
\end{tabular}




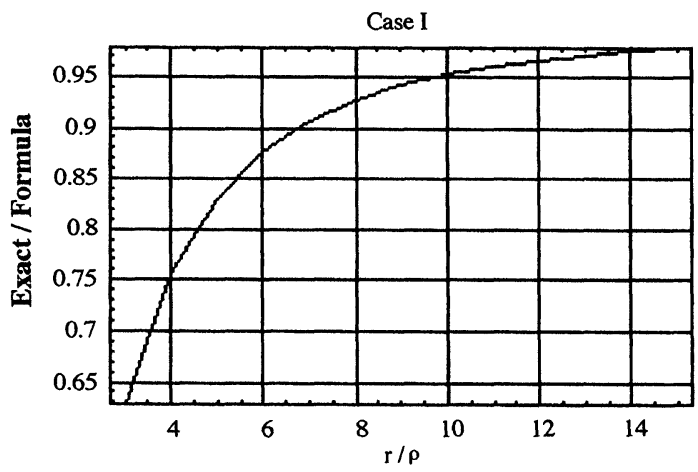

FIGURE 3 Ratio of exact over formula forces for case I.

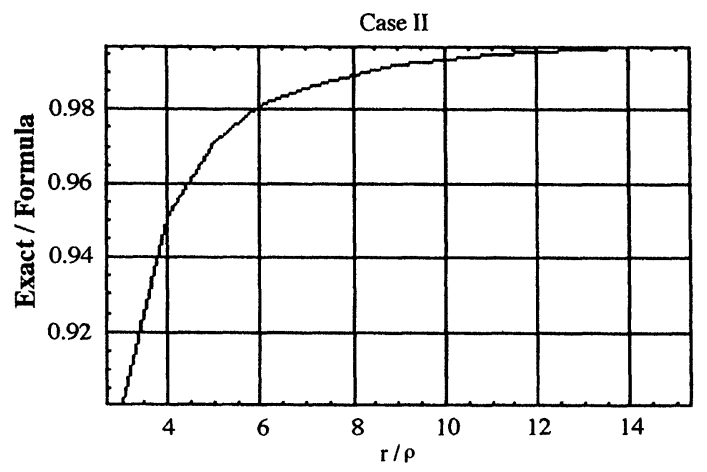

FIGURE 4 Ratio of exact over formula forces for case II.

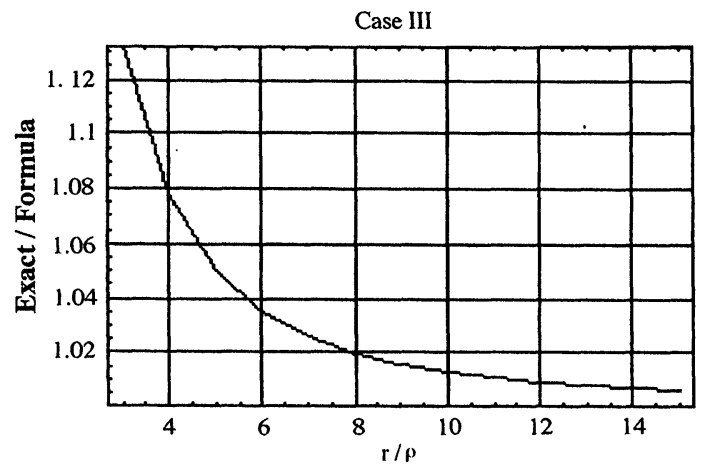

FIGURE 5 Ratio of exact over formula forces for case III. 


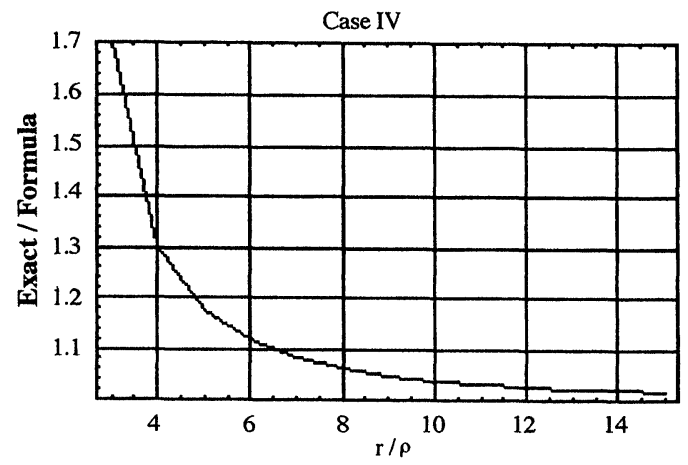

FIGURE 6 Ratio of exact over formula forces for case IV.

\section{CONCLUSIONS}

We derived the analytical expression of the force between two magnetic dipoles using both the path integral and vector differentiation methods, and get the same result.

\section{References}

[1] J. Greene and F.G. Karioris, "Force on a magnetic dipole", Am. J. Phys. 39, 172-175 (1970).

[2] L. Vaidman, "Torque and force on a magnetic dipole", Am. J. Phys. 58, 978-983 (1990).

[3] T.H. Boyer, "The force on a magnetic dipole" Am. J. Phys. 56, 688-692 (1988).

[4] K.R. Brownstein, "Force exerted on a magnetic dipole", Am. J. Phys. 61, 940-941 (1993).

[5] P. Hrasko, "Forces acting on magnetic dipoles", II Nuovo Cimento 3B, 213-224 (1971).

[6] V. Hnizdo, "Hidden momentum and the force on a magnetic dipole", Magnetic and Electrical Separation 3, 259-265 (1992).

[7] P. Lorrain and D.R. Corson, Electromagnetic Fields and Waves (W.H. Freeman and Company, NY, 1970), p. 322.

[8] P.B. Landecker, D.D. Villani and K.W. Yung, "An analytic solution for the torque between two magnetic dipoles", in preparation. 


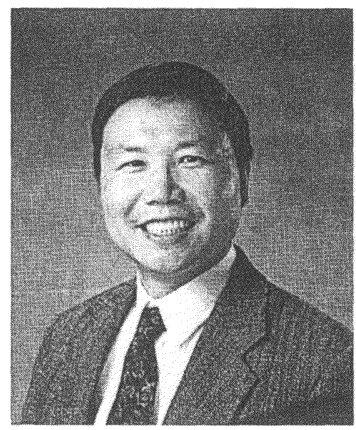

Kar W. Yung obtained his B.S. and M.S. in Physics 1973, and Ph.D. in Physics, 1980, all from California Institute of Technology, Pasadena, CA, USA. He is employed as a scientist at the Hughes Space and Communication Company, El Segundo, CA, USA. Dr. Yung worked on system engineering, project management, system simulation and analysis tasks for various programs. He has experience with $\mathrm{Al}$ Bayesian Network, resource management, satellite mission, coverage and loading analyses, reliability and replenishment analyses, radar SAR processing and image processing. $\mathrm{He}$ is also familiar with software engineering and development techniques. Dr. Yung has patents in antenna design, navigation and positioning systems, constellation design, digitization techniques and synthetic aperture correlators.

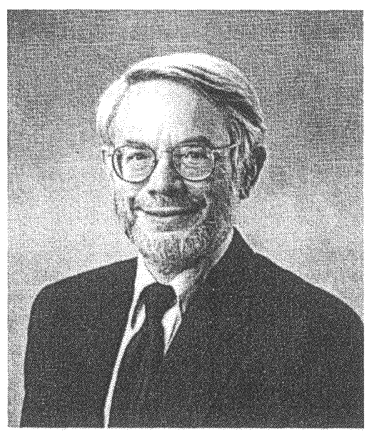

Peter B. Landecker obtained his B.S. in Physics, 1963 from Columbia University, New York, NY, USA, and Ph.D. in Physics, 1968, from Cornell University, Ithaca, NY, USA. Dr. Landecker is a senior scientist with Hughes Space and Communication Company, El Segundo, CA, USA. He specializes in design, development, integration and testing of remote sensing spacecraft payloads. Previously he was the principal investigator of solar X-ray spectrometer/ spectroheliograph that flew on P78-1 spacecraft. Dr. Landecker worked on NASA Orbiting Solar Observatory and Skylab programs. He built and operated the world's deepest neutrino detector in South Africa. $\mathrm{He}$ has published over 70 papers and received six awards. He also has patents in autonomous spacecraft attitude and ephemeris and stabilizing spacecraft camera pointing.

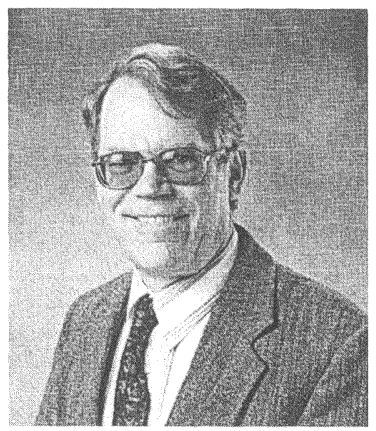

Daniel D. Villani obtained his B.S. in Physics, 1969, from California Institute of Technology, Pasadena, CA, USA, and M.A. and Ph.D. in Engineering, 1983, from Princeton University, Princeton, NJ, USA. Dr. Villani is the project manager, Mission Systems Engineering and Operations, Hughes Space and Communications Company, El Segundo, CA, USA. He participated in Pioneer Venus, and was a Payload Systems Engineer on GOES-5/6, GMS-2/3, Deputy Radar systems Engineering Team Chief, Magellan Radar, analyst on Galileo Probe team and Mission System Engineer/Flight Director for several commercial communications satellites including the HGS-1 lunar swingby. Dr. Villani received NASA Public Service Medal for Magellan Radar Mission. 